\title{
Clear-cell Renal Cell Carcinoma: Molecular Characterization of IMDC Risk Groups and Sarcomatoid Tumors
}

\author{
Annelies Verbiest, ${ }^{1,2}$ Inne Renders, ${ }^{1}$ Stefano Caruso, ${ }^{3}$ Gabrielle Couchy, ${ }^{3}$ \\ Sylvie Job, ${ }^{4}$ Annouschka Laenen, ${ }^{5}$ Virginie Verkarre, ${ }^{6}$ Nathalie Rioux-Leclercq, ${ }^{7}$ \\ Patrick Schöffski, ${ }^{1,2}$ Yann Vano, ${ }^{8}$ Reza-Thierry Elaidi, ${ }^{8}$ Evelyne Lerut, ${ }^{9}$ \\ Maarten Albersen, ${ }^{10}$ Stéphane Oudard, ${ }^{8}$ Wolf-Hervé Fridman, ${ }^{11}$ \\ Catherine Sautès-Fridman, ${ }^{11}$ Laurence Albigès, ${ }^{12}$ Agnieszka Wozniak, ${ }^{2}$ \\ Jessica Zucman-Rossi, ${ }^{3}$ Benoit Beuselinck ${ }^{1,2,3}$
}

\begin{abstract}
In advanced clear-cell renal cell carcinoma, the Checkmate214 study showed superior outcomes with ipilimumab-nivolumab in International Metastatic RCC Database Consortium intermediate-/poor-risk patients and with sunitinib in good-risk patients. We demonstrate how underlying molecular subtypes and angiogenic gene expression can explain these differences and can characterize the heterogeneous International Metastatic RCC Database Consortium intermediate-risk group.

Introduction: Recent trials have suggested predictive biomarkers in advanced clear-cell renal cell carcinoma (accRCC): International Metastatic RCC Database Consortium (IMDC) good risk or angiogenic gene signature for sunitinib and IMDC intermediate/poor risk for ipilimumab-nivolumab and T-effector cell signature or sarcomatoid dedifferentiation for atezolizumab-bevacizumab. We hypothesized that earlier described molecular subtypes, ccrcc1 to ccrcc4, could provide similar information as a single generic biomarker and molecularly characterize the heterogeneous intermediate-risk group. Patients and Methods: Patients with accRCC treated with systemic therapies were included. We assessed associations between the 5 biomarkers and their impact on progression-free survival (PFS) and response rate (RR) on first-line sunitinib or pazopanib. The cutoff percentage of sarcomatoid dedifferentiation with optimal discriminative value was determined. Results: In total, 430 patients were included (163 with molecular data). The molecular ccrcc2 subtype identified tumors with higher angiogenic gene expression across IMDC risk groups: prevalence was high in IMDC good risk and low in IMDC poor risk $(P<.001)$. Molecular subtype, IMDC, and angiogenic gene expression had comparable C-indices to predict PFS and RR (range, $60 \%-66 \%$ ). The ccrcc2 subtype and angiogenic gene expression were positive predictors of PFS in IMDC intermediate-risk patients $(P=.006$; $P=.04)$. Immune signature did not differ between IMDC groups, but was strongly correlated with molecular subtype $(P=.8$ and $P=.0007)$. A cutoff value of $25 \%$ sarcomatoid differentiation discriminated tumors with distinct molecular characteristics and therapeutic sensitivity. Conclusion: In accRCC, molecular subtypes can explain differences in IMDC risk group, expression of angiogenesis and immune response genes, and sarcomatoid dedifferentiation. They
\end{abstract}

Abstract

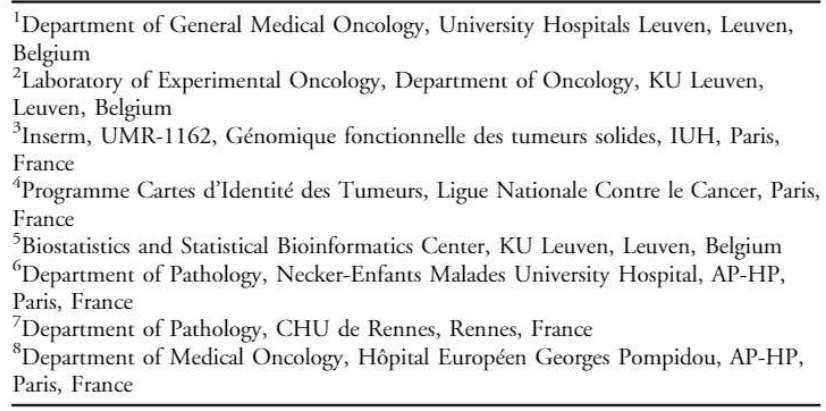

${ }_{9}^{9}$ Department of Imaging and Pathology, KU Leuven, Leuven, Belgium
${ }^{10}$ Department of Urology, University Hospitals Leuven, Leuven, Belgium
${ }^{11}$ Inserm, UMR-S1138, Centre de Recherche des Cordeliers, Sorbonne University,
Paris, France
${ }^{12}$ Department of Medical Oncology, Institut Gustave Roussy, Villejuif, France

Submitted: Mar 7, 2019; Revised: Apr 24, 2019; Accepted: May 20, 2019

Address for correspondence: Benoit Beuselinck, MD, Department of General Medical Oncology, University Hospitals Leuven, Herestraat 49, 3000 Leuven, Belgium E-mail contact: benoit.beuselinck@uzleuven.be 
ARTICLE IN PRESS

\section{Molecular Subtypes and Sarcomatoid Features in Clear-cell RCC}

can identify molecularly different patient populations within the heterogeneous IMDC intermediate group and select patients for systemic therapies.

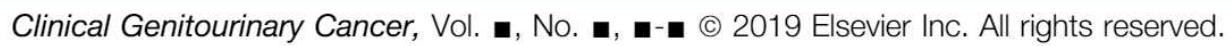

Keywords: Angiogenesis, Biomarker, Immune checkpoint inhibitor, Molecular subtype, Sunitinib

\section{Introduction}

Clear-cell renal cell carcinomas (ccRCCs) are hallmarked by hypervascularity on the one hand and their capacity to elicit an immune response on the other hand. ${ }^{1}$ Advanced disease (accRCC) may therefore be treated with angiogenesis inhibitors, immune checkpoint inhibitors (ICPIs), or a combination strategy. ${ }^{2-7}$ Outcomes on different therapies are, however, highly variable, and in the absence of predictive biomarkers, the optimal way of patient selection is unclear.

Only very recently have some advances in the field of predictive biomarkers been made. The phase III Checkmate214 trial (NCT0221749) compared ipilimumab-nivolumab with the angiogenesis inhibitor sunitinib in first-line therapy, after stratification for International Metastatic RCC Database Consortium (IMDC) risk group. ${ }^{7,8}$ Ipilimumab-nivolumab showed efficacy across all IMDC risk groups and was superior over sunitinib in intermediate-/poorrisk patients. It is therefore recommended in this population. Sunitinib, on the other hand, yielded favorable outcomes in goodrisk patients, and sunitinib or pazopanib are recommended for these patients. ${ }^{2}$ However, the IMDC risk group has important limitations as a biomarker. It is based on clinical risk factors that may change over time, and the majority of patients are intermediate-risk, a group that is known to be very heterogeneous in terms of prognosis and treatment outcome..$^{8-10}$

The phase III IMmotion151 trial (NCT02420821) compared atezolizumab-bevacizumab with sunitinib in first-line treatment and validated predictive gene signatures. ${ }^{4,11}$ A T-effector cell ( $\mathrm{T}_{\text {eff }}$ ) signature and programmed death ligand 1 (PD-L1) expression were associated with favorable outcomes on atezolizumab-bevacizumab and were similar across risk groups. An angiogenic gene signature was associated with favorable outcomes on sunitinib, and was higher in good-risk patients. Patients with sarcomatoid tumors responded poorly to sunitinib and had lower angiogenic signatures, but higher $\mathrm{T}_{\text {eff-signatures and PD-L1 expression. Yet, although these signatures }}$ are very promising, they have been developed specifically for sunitinib and atezolizumab-bevacizumab. Therefore, they only partly reflect intrinsic tumor biology and cannot be extrapolated to other treatments.

Another predictive marker that has been described in accRCC are 4 molecular subtypes, ccrccl to ccrcc4. ${ }^{12}$ These subtypes were established based on unsupervised clustering of whole genome mRNA-expression data, and therefore reflect intrinsic differences in

Table 1 Correlation of Different Biomarkers With Outeome on First-line Sunitinib or Pazopanib

\begin{tabular}{|c|c|c|c|c|c|c|c|c|c|}
\hline & N & PFS, mos & $P$ & $\begin{array}{l}\text { C-index } \\
(95 \% \text { CI) }\end{array}$ & RR, \% & $P$ & $\begin{array}{l}\text { C-index } \\
\text { (95\% Cl) }\end{array}$ & OS, mos & $P$ \\
\hline IMDC & 145 & & & & & & & & \\
\hline Good & 21 & 25 & .0002 & $\begin{array}{c}0.60 \\
(0.55-0.65)\end{array}$ & 63 & .01 & $\begin{array}{c}0.62 \\
(0.54-0.70)\end{array}$ & 72 & $<.0001$ \\
\hline Intermediate & 95 & 13 & & & 46 & & & 24 & \\
\hline Poor & 29 & 5 & & & 19 & & & 12 & \\
\hline ccrcc1-4 subtype & 145 & & & & & & & & \\
\hline $\operatorname{ccrcc} 1$ & 42 & 12 & $<.0001$ & $\begin{array}{c}0.60 \\
(0.57-0.68)\end{array}$ & 34 & .01 & $\begin{array}{c}0.66 \\
(0.57-0.75)\end{array}$ & 23 & .0002 \\
\hline ccrcc2 & 63 & 19 & & & 57 & & & 31 & \\
\hline ccrcc3 & 9 & 10 & & & 63 & & & 50 & \\
\hline ccrcc4 & 31 & 5 & & & 25 & & & 11 & \\
\hline Angio score & 145 & & & & & & & & \\
\hline High & 74 & 19 & $<.0001$ & $\begin{array}{c}0.64 \\
(0.58-0.69)\end{array}$ & 56 & .01 & $\begin{array}{c}0.60 \\
(0.50-0.70)\end{array}$ & 28 & .005 \\
\hline Low & 71 & 8 & & & 33 & & & 19 & \\
\hline Sarcomatoid & 366 & & & & & & & & \\
\hline$<25 \%$ & 344 & 11 & $<.0001$ & & 46 & .001 & & 24 & $<.0001$ \\
\hline$\geq 25 \%$ & 22 & 3 & & & 10 & & & 8 & \\
\hline
\end{tabular}

The discriminative power of predictors of RR and PFS was quantified with Harrells C-index (Harrell et al, ${ }^{18}$ JAMA 1982).

Favorable IMDC risk group, ccrcc2 or ccrcc3 molecular subtype, High Angio score, and $<25 \%$ sarcomatoid dedifferentation are predictors of favorable outcome.

Abbreviations: $\mathrm{Cl}=$ Confidence interval; IMDC = International Metastatic RCC Database Consortium; $\mathrm{N}=$ number; $\mathrm{OS}=$ overall survival; $\mathrm{PFS}=$ median progression-free survival; RR = response rate. 


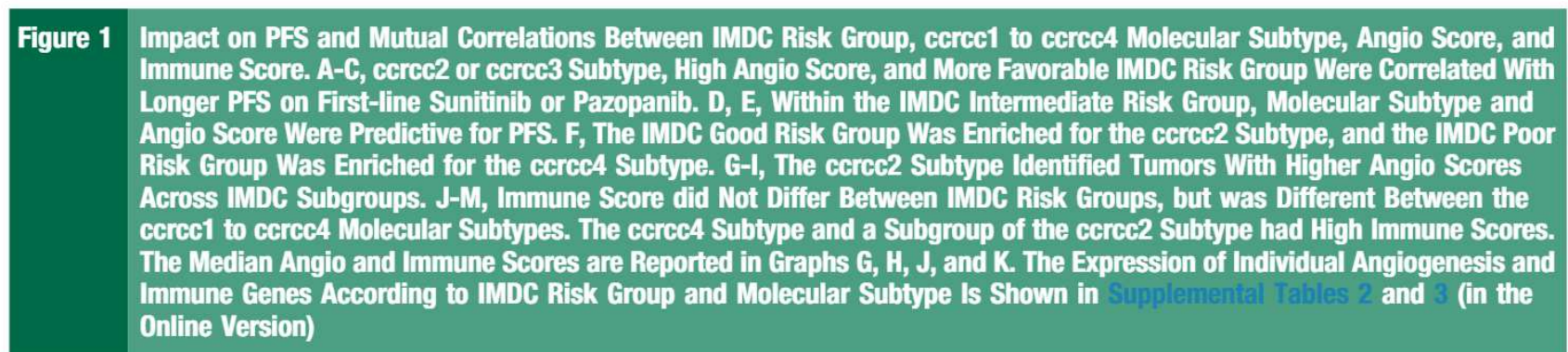

Progression free survival on first-line sunitinib or pazopanib

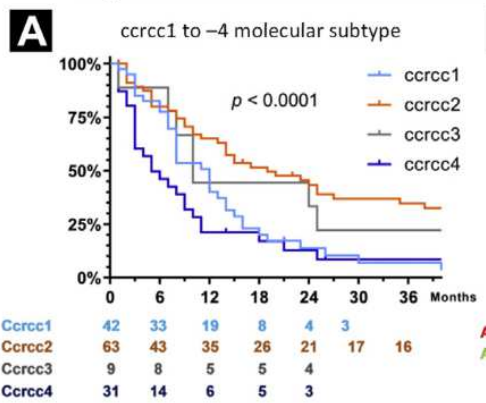

B

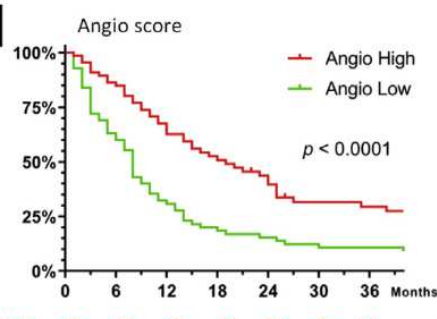

Angio High $\quad \begin{array}{lllllll}71 & 56 & 42 & 30 & 22 & 15 & 14\end{array}$

Progression free survival on first-line sunitinib or pazopanib within IMDC Intermediate risk
D

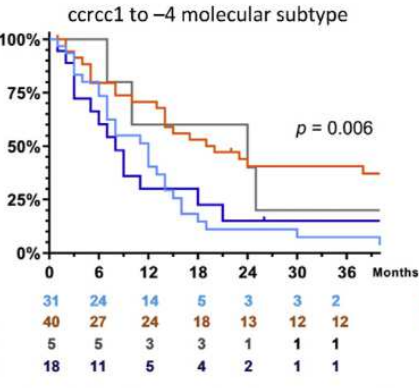

$\mathbf{G}$

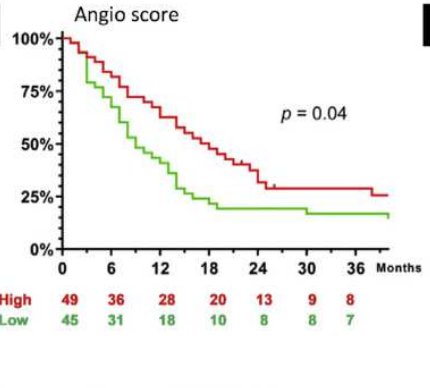

$\mathbf{H}$
$\mathbf{G}$
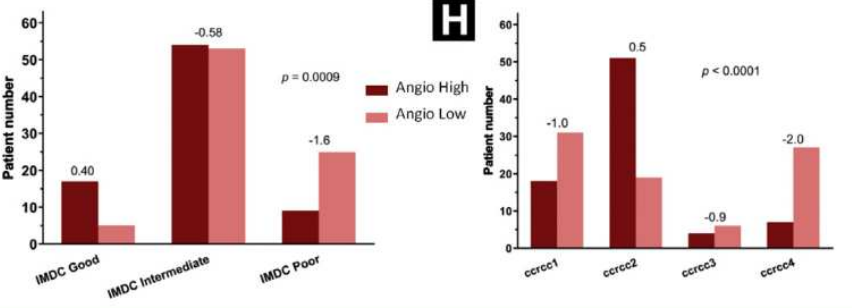
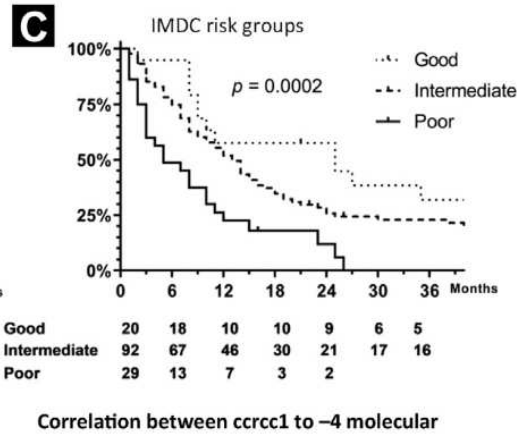

subtype and IMDC risk group

$\mathbf{F}$

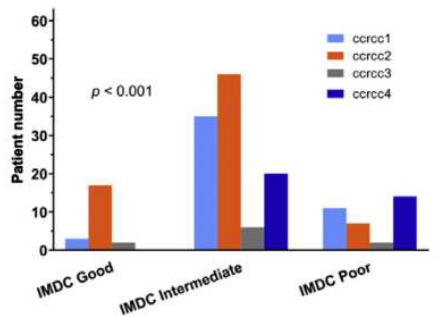

Bivariable Cox regression of IMDC and ccrcc1 to -4 molecular subtype for impact on Angio score:

IMDC $\quad p=0.13$

ccrec1 to $-4 \quad p<0.0001$

Abbreviations: IMDC = International Metastatic RCC Database Consortium; PFS $=$ progression-free survival.

tumor biology. They also differ in terms of immune infiltrate, cytogenetic anomalies, sarcomatoid dedifferentiation, methylation, and mutation profile in the tumor. The subtypes are prognostic for overall survival (OS) after nephrectomy and after metastasectomy with curative intent. ${ }^{12,13}$ The prognostic value of similar subtypes has been described by others in the setting of nephrectomy with curative intent. ${ }^{14,15}$ However, the ccrcc1 to ccrcc4 subtypes are also predictive for outcome on first-line sunitinib or pazopanib. ${ }^{12,16}$ ccrcc 2 tumors are characterized by pro-angiogenic gene expression and ccrcc3 tumors by a normal-kidney-like gene expression pattern. ${ }^{12,17}$ Both ccrcc2 and ccrcc 3 have a good prognosis and favorable outcomes with sunitinib or pazopanib. A subset of ccrcc2 tumors also exhibit an active immune response. The 2 other subtypes, ccrcc1 and ccrcc4, are marked by upregulation of MYCtargets. ccrcc1 tumors have an intermediate prognosis and outcome on sunitinib or pazopanib and an immune cold pheno-

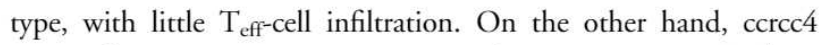
tumors have a very poor prognosis and outcome on sunitinib or pazopanib, but are marked by a strongly inflamed, yet exhausted immune phenotype and are often sarcomatoid.

We hypothesized that the ccrcc1 to ccrcc4 molecular subtypes can explain differences in IMDC risk group, angiogenic signature, $\mathrm{T}_{\text {eff }}$-signature, and sarcomatoid dedifferentiation, thus providing a maximum of information as a single biomarker. Therefore, the 


\section{Molecular Subtypes and Sarcomatoid Features in Clear-cell RCC}

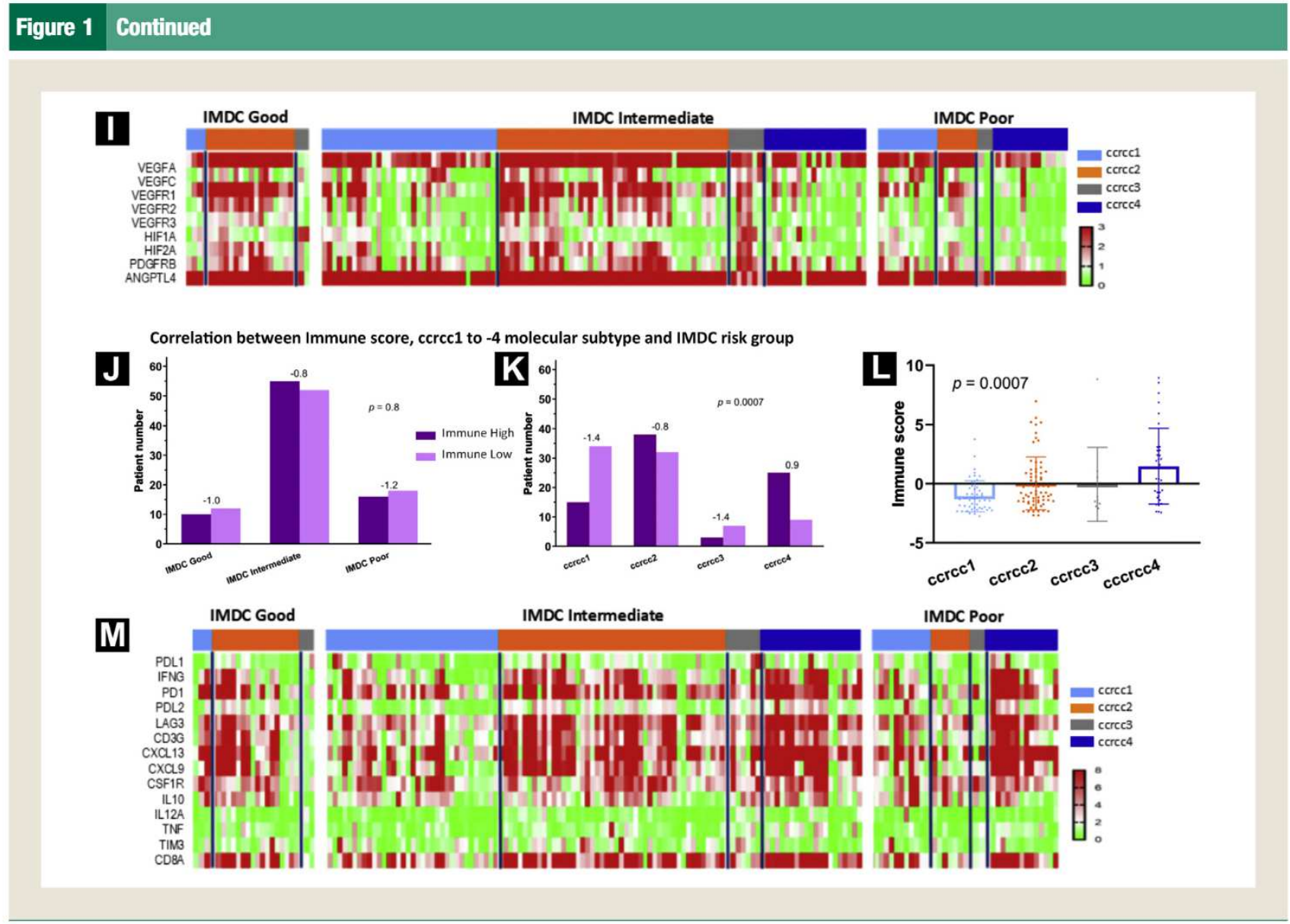

major aims of this study were to assess molecular differences between tumors in function of IMDC risk group and sarcomatoid dedifferentiation and correlate these with outcome on first-line sunitinib or pazopanib. A secondary aim was to characterize the heterogeneous IMDC intermediate-risk group on a molecular level.

\section{Patients and Methods Patients and Tissue}

The CIT-rein database was searched for patients of whom the clinical and molecular data addressed in this study were available. The CIT-rein database is a French-Belgian multicenter database that includes patients with accRCC treated with systemic therapy or metastasectomy, containing over 600 cases. Patients are included after informed consent, and both clinical data and tumoral tissue are collected in a prospective way. Primary tumor tissue is collected at time of nephrectomy, before the start of any systemic therapy. Clear-cell histology and sarcomatoid dedifferentiation are confirmed by an expert uro-pathologist (N.R.L., E.L., V.V.). Outcome is systematically measured with Response Evaluation Criteria In Solid Tumors (RECIST) by one of the authors (B.B.). Drugs are dosed according to their manufacturer's label, with dose reductions as needed. Timing of assessments is at the discretion of the treating physician, usually once every 3 months. The study was approved by the ethics committees of the partaking centers.

\section{Molecular Profiling}

In patients from CIT-rein from whom fresh frozen primary tumor is available, expression of 98 genes is routinely determined with a custom panel (Fluidigm). This panel contains, among others, genes involved in angiogenesis and immune response. Molecular subtype is determined with an established 35-gene classifier algorithm as described previously. ${ }^{12}$

Angiogenic gene expression was quantified using 9 available genes involved in vascular endothelial growth factor (VEGF)-dependent angiogenesis. Fold changes were summarized as one "Angio score," using principal component analysis. Samples were also labeled as "Angio high" or "Angio low," depending on whether their Angio score was above or equal/below the median. Similarly, an "Immune score" was calculated using 13 available genes involved in immune response. As all genes are part of a routine panel, these scores were not trained as predictors for treatment outcome, nor is the Immune score an optimal signature of $\mathrm{T}_{\text {eff-cells. }}$.

\section{Statistical Analysis}

The optimal cutoff value for sarcomatoid dedifferentiation in order to predict progression-free survival (PFS) and overall survival (OS) was determined after considering all possible cutoffs in a Cox proportional hazard model, and selecting the dichomatization leading to the highest likelihood. 


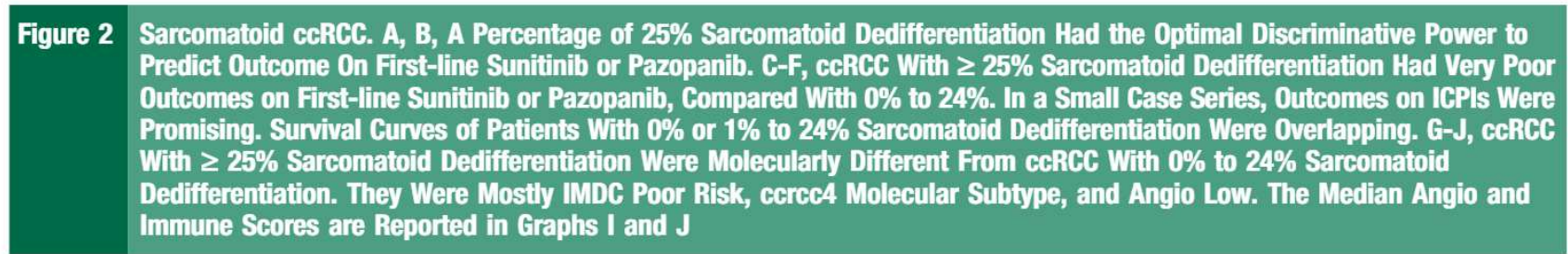

Establishing $\mathbf{2 5 \%}$ as the optimal cut-off percentage for sarcomatoid dedifferentiation
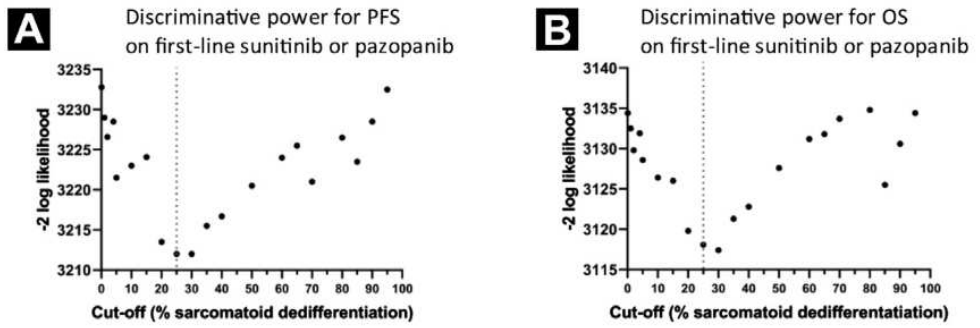

Outcome of 366 ccRCC with $0 \%, 1-24 \%$ or $\geq 25 \%$ sarcomatoid dedifferentiation on first-line sunitinib or pazopanib, and of 92 patients on immune checkpoint inhibitors ( 4 with $\geq 25 \%$ sarcomatoid dedifferentiation)
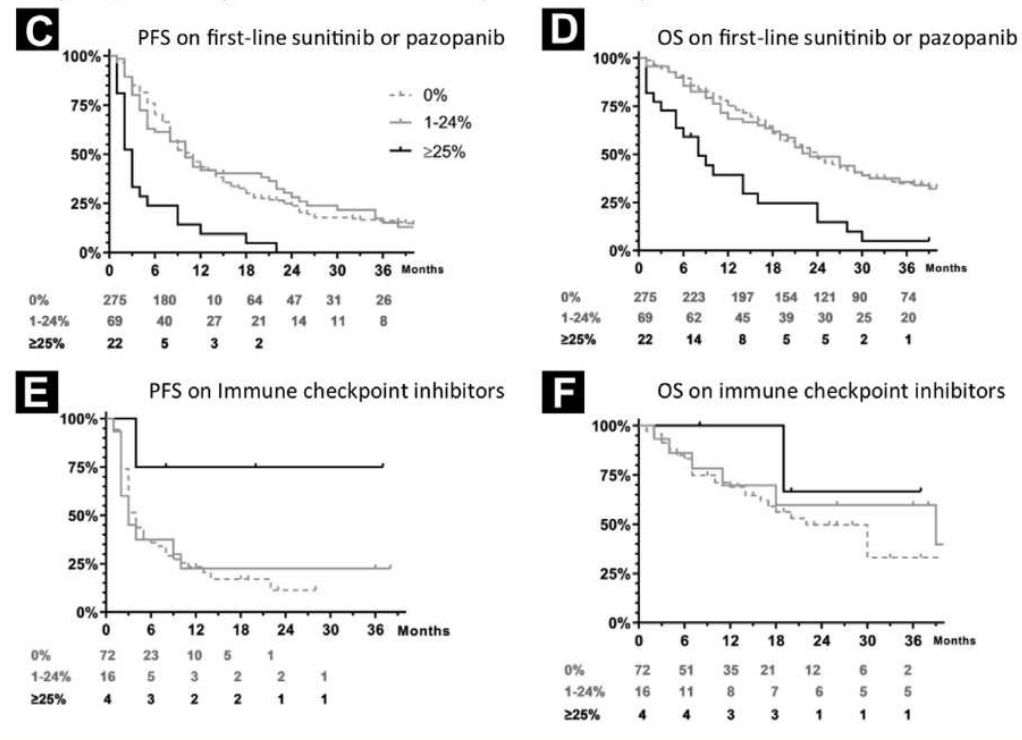

Abbreviations: $\mathrm{ccRCC}=$ clear-cell renal cell carcinoma; ICPIS = immune checkpoint Inhibitors; IMDC = International RCC Database Consortium; OS = overall survival; PFS = progression-free survival.

To assess the predictive impact of different biomarkers, we focused on their impact on response rate (RR) and PFS, as OS is heavily influenced by prognostic factors and subsequent therapies. The association of predictors with RR was analyzed using logistic regression. Their association with PFS was analyzed with Cox proportional hazards regression.

The Kruskal-Wallis and Mann-Whitney $U$ tests were used to compare expression of individual genes between IMDC groups, molecular subtypes, and sarcomatoid yes/no. The associations between IMDC risk groups, molecular subtype, Angio high/low, Immune high/low, and sarcomatoid yes/no were tested with the Fisher exact test.
Analyses were done using SAS 9.4, GraphPad Prism 7.2, and XLStat 2018.7.

\section{Results}

\section{Molecular Differences Between IMDC Risk Groups}

Molecular data and IMDC risk group were available for 163 patients, 145 of whom were treated with first-line sunitinib or pazopanib (see Supplemental Table 1 in the online version).

A more favorable IMDC risk group, a ccrcc2 or ccrcc3 molecular subtype, and higher Angio score were all positive predictors for longer PFS and higher RR on first-line sunitinib or pazopanib (Table 1; Figure 1). The discriminative value of these predictors, 
ARTICLE IN PRESS

\section{Molecular Subtypes and Sarcomatoid Features in Clear-cell RCC}

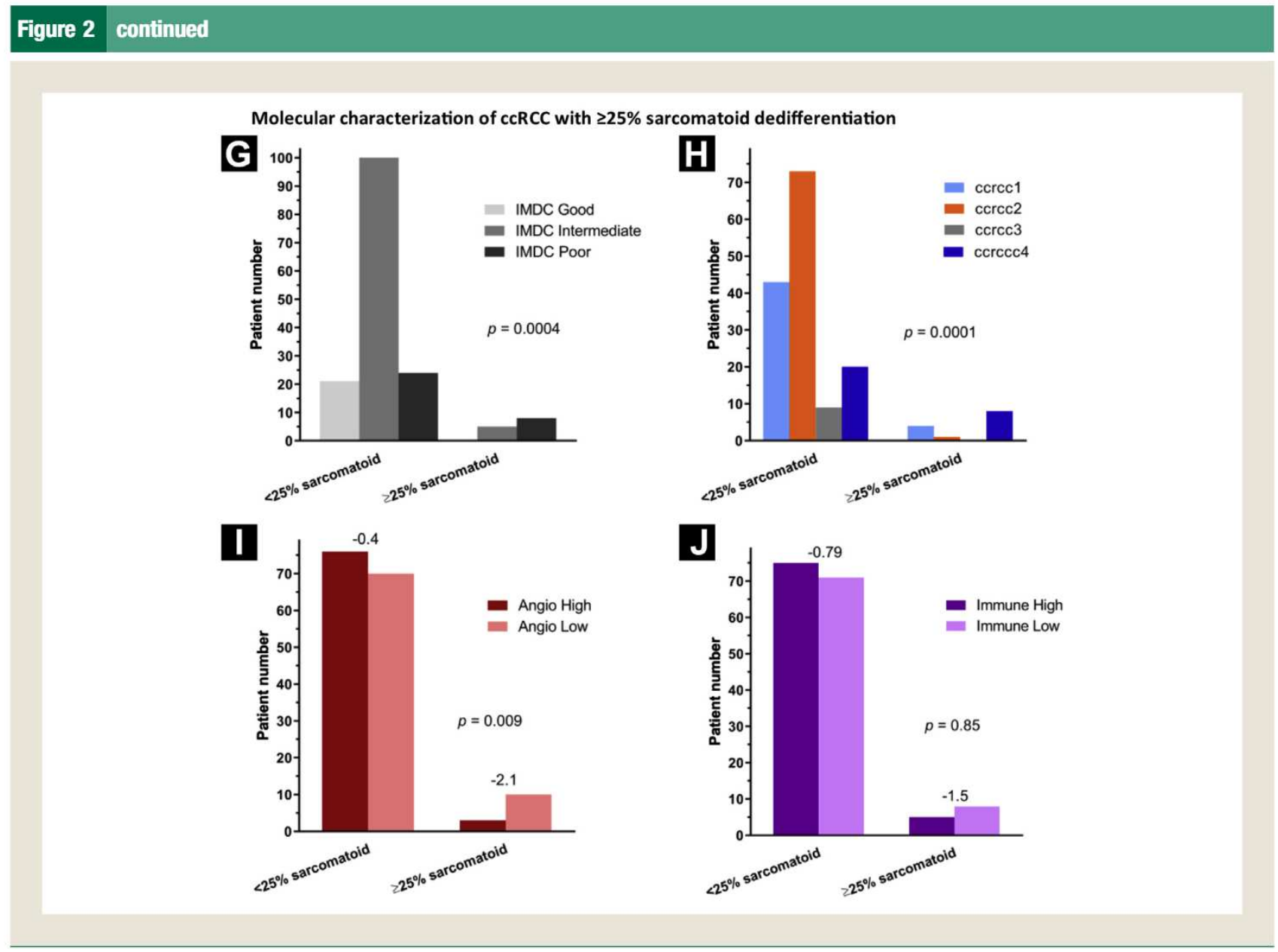

quantified by their C-index, was comparable. The combination of markers did not result in a meaningful increase in discriminative value.

IMDC risk group, molecular subtype, and Angio score were strongly associated with each other (Figure 1). The pro-angiogenic ccrcc2 molecular subtype could identify patients with higher Angio scores across IMDC risk groups. The IMDC good-risk group was enriched for the ccrcc2 subtype and Angio-high tumors (both $77 \% ; P<.001)$. The IMDC poor-risk group was enriched for the unfavorable ccrcc4 subtype (41\%) and Angio-low tumors (74\%). The IMDC intermediate-risk group was heterogeneous, with a normal distribution of the molecular subtypes ( $46 \%$ ccrcc 2$)$ and $50 \%$ Angio-high and -low tumors. The expression of specific targets of sunitinib and pazopanib (VEGFR1, -2, -3) was highest in IMDC good-risk patients and lowest in IMDC poor-risk patients (see Supplemental Table 2 in the online version). Within the intermediate-risk group, the ccrcc 2 subtype could identify tumors with higher angiogenic gene expression. Indeed, upon bivariable analysis, the differences in Angio score between IMDC risk groups became non-significant after correction for molecular subtype. Within the IMDC intermediate-risk group, both molecular subtype and Angio score were predictive for PFS.

The expression of genes related to immune response, summarized by the Immune score, did not differ between IMDC risk groups
$(P=.7)$. Contrarily, the differences in the Immune score between molecular subtypes were highly significant $(P=.0007)$. ccrcc4 tumors, and a subgroup of ccrcc 2 tumors, were marked by high Immune scores (Figure 1; Supplemental Table 3 in the online version). Thus, molecular subtypes could identify tumors with high immune gene expression across IMDC risk groups.

\section{Sarcomatoid ccRCC}

The impact of sarcomatoid dedifferentiation was tested in 366 patients treated with first-line sunitinib or pazopanib (see Supplemental Table 4 in the online version). The optimal cutoff associated with PFS and OS was $\geq 25 \%$ sarcomatoid dedifferentiation. PFS and OS curves for patients with $0 \%$ or $1 \%$ to $24 \%$ sarcomatoid dedifferentiation were overlapping (Figure 2). On multivariable analysis, sarcomatoid dedifferentiation $\geq 25 \%$ was independently associated with PFS (hazard ratio, 2.9; 95\% confidence interval, 1.8-4.5) and OS (hazard ratio, 2.3; 95\% confidence interval, 1.4-3.7) (see Supplemental Tables 5 and 6 in the online version). For tumors with $0 \%$ to $24 \%$ versus $\geq 25 \%$ sarcomatoid dedifferentiation, RR was $46 \%$ versus $10 \%(P=.001)$, and PFS was 11 months versus 3 months $(P<.0001)$ (Table 1$)$.

Molecular differences and IMDC risk group were tested in 163 patients. Compared with tumors with $0 \%$ to $24 \%$ sarcomatoid dedifferentiation, tumors with $\geq 25 \%$ dedifferentiation were 


\section{Annelies Verbiest et al}

typically IMDC poor risk $(62 \% ; P=.0004)$ and ccrcc4 subtype $(62 \% ; P=.0001)$. They had lower Angio scores $(P=.009)$ but no difference in Immune scores $(P=.35)$. Tumors with $0 \%$ versus $1 \%$ to $24 \%$ sarcomatoid dedifferentiation had similar IMDC risk groups and Angio scores (see Supplemental Tables 7 and 8 in the online version).

In an exploratory analysis, we assessed outcomes of 92 patients treated with ICPI, mostly in the second- or a consecutive line. The 4 tumors with $\geq 25 \%$ sarcomatoid dedifferentiation all responded, whereas RR in 88 others was only $16 \%$. PFS curves for $0 \%$ and $1 \%$ to $24 \%$ sarcomatoid dedifferentiation were overlapping and inferior to those for $\geq 25 \%$.

\section{Pooling of IMDC Intermediate and Poor Risk Groups}

As phase III trials have typically reported outcomes of pooled IMDC intermediate-/poor- versus good-risk patients, we explicitly assessed differences between the intermediate- and poor-risk groups. ${ }^{3,7}$ Outcomes on first-line sunitinib or pazopanib, distribution of molecular subtypes, Angio score, and proportion of $\geq 25 \%$ sarcomatoid tumors were all significantly different between the intermediate- and poor-risk groups. Moreover, when we repeated all analyses while comparing IMDC good risk with pooled intermediate/poor risk, the results were not as solid as when considering the 3 groups separately (see Supplemental Tables 9 and 10 in the online version).

\section{Discussion}

There is still no optimal way of selecting patients with accRCC for treatment with angiogenesis inhibitors, ICPIs, or the upcoming combinations. Recent trials have suggested IMDC risk group, angiogenic or $T_{\text {eff }}$ gene expression, and sarcomatoid dedifferentiation as possible biomarkers, but they all have important limitations. ${ }^{4,7,11}$ In this study, we assessed the correlations between these markers and investigated whether the information they provide could be summarized by a single biomarker, the molecular ccrcc1 to ccrcc4 subtypes. A secondary aim was to characterize the large but heterogeneous IMDC intermediate-risk group on a molecular level.

IMDC good-risk patients had higher angiogenic gene expression, associated with response to sunitinib. This was explained by the overrepresentation of the pro-angiogenic ccrcc 2 subtype in the IMDC good-risk group. The predictive value for outcome on sunitinib or pazopanib was similar for molecular subtypes, IMDC risk groups, and angiogenic signature. But more importantly, the ccrcc2 subtype could identify tumors with an angiogenic signature within the IMDC intermediate-risk group and was predictive for outcome on sunitinib and pazopanib in this population. As the majority of patients with accRCC are IMDC intermediate risk, molecular subtypes could contribute to personalized therapy decisions in this population.

When looking at immune gene expression, we found no differences between IMDC risk groups. This is in accordance with responses to ICPIs that were observed across all risk groups in the Checkmate214

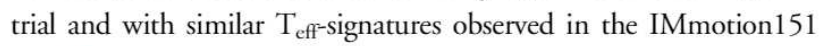
trial. ${ }^{4,7}$ However, molecular subtypes showed marked differences in immune gene expression, with ccrcc 4 and a subgroup of ccrcc 2 tumors displaying immune activation. Therefore, the lack of difference in Immune score between IMDC risk groups hides more pronounced differences in immune phenotype. IMDC good-risk patients mainly have ccrcc 2 tumors, a subgroup of which have an activated immune response. IMDC poor-risk patients mainly have either immuneexhausted ccrcc 4 tumors or immune-cold ccrccl tumors. ${ }^{12}$

We also found a marked difference between IMDC intermediateand poor-risk patients on a molecular level. Any comparison of these risk groups should therefore be performed preferentially without pooling IMDC intermediate- and poor-risk patients.

Based on these data, ccrcc1 to ccrcc 4 molecular subtypes incorporate in a single marker the information provided by IMDC risk group and expression of angiogenesis and immune response genes. The subtypes were not developed as a marker for a specific therapy and might therefore retain their value in different therapeutic settings and for the development of novel therapeutic options. For example, in the setting of metastasectomy, ccrcc1 and ccrcc4 tumors are at risk for early relapse. ${ }^{13}$ Upon treatment with sunitinib or pazopanib, ccrcc2 and ccrcc 3 have favorable outcomes. ccrcc 4 tumors can be expected to be the best candidates for ICPIs. As earlier research showed that ccrcc 4 tumors exhibit an immune suppressive microenvironment with high levels of myeloid inflammation, they will probably benefit especially from a combination of ICPIs and angiogenesis inhibitor, where the latter suppresses myeloid inflammation. ${ }^{12}$ For the immune-cold ccrcc1 tumors, immune sensitizing therapies should be investigated.

Sarcomatoid dedifferentiation has been associated with poor prognosis and outcome on sunitinib or pazopanib. ${ }^{19,20}$ We showed on a large patient series that a cutoff percentage of $25 \%$ sarcomatoid dedifferentiation separated tumors with distinct therapeutic sensitivity and molecular characteristics. Tumors with $\geq 25 \%$ sarcomatoid dedifferentiation had poor outcomes on first-line sunitinib or pazopanib, low angiogenic gene expression, and were typically IMDC poor risk and ccrcc4 subtype. Contrarily, outcomes and molecular characteristics were similar for tumors with $0 \%$ versus $1 \%$ to $24 \%$ sarcomatoid dedifferentiation. Interestingly, 4 sarcomatoid tumors treated with ICPIs at our institution all responded, whereas outcomes were poorer in 88 patients with $0 \%$ to $24 \%$ sarcomatoid dedifferentiation.

This study has its limitations. Most importantly, the CIT database only contains a limited number of patients treated with ICPIs, to assess the hypothesized predictive value of molecular subtypes in this context. Two ongoing prospective trials are evaluating this question (Nivoren NCT03013335; Bionikk NCT02960906). Secondly, molecular subtype is currently determined on fresh frozen tissue. To facilitate the classification procedure, a major future aim is to validate the classifier on paraffin-embedded tissue.

In conclusion, molecular subtypes in accRCC can explain differences in IMDC risk group, expression of angiogenic and immune response genes, and sarcomatoid dedifferentation. Favorable outcomes on first-line sunitinib or pazopanib are predicted by IMDC good risk group, ccrcc2 molecular subtype, and angiogenic gene expression. Molecular subtypes could select patients within the IMDC intermediate risk group for angiogenesis inhibitors, but validation on paraffinembedded tissue is needed before potential clinical implementation. As molecular subtypes also identify tumors with different immune profiles, where IMDC risk groups do not, they have a potential use in the setting of ICPIs and combination therapies. For sarcomatoid dedifferentiaton, a cutoff value of $25 \%$ separates tumors with distinct prognosis and molecular characteristics. Tumors with $\geq 25 \%$ sarcomatoid dedifferentiation have low angiogenic gene expression and are 


\section{Molecular Subtypes and Sarcomatoid Features in Clear-cell RCC}

typically IMDC poor risk and ccrcc4 subtype. They have poor outcomes on sunitinib or pazopanib, but ICPIs seem a promising option.

\section{Clinical Practice Points}

- Metastatic RCC can be treated with angiogenesis inhibitors, ICPIs, or combination therapy. There is currently no reliable way of patient selection. IMDC good risk is associated with better outcome on sunitinib, whereas ICPIs are effective across IMDC risk groups. The majority of patients are intermediate risk, with very heterogeneous outcomes on all therapies.

- ccRCC can be divided into 4 molecular subtypes, ccrcc1 to ccrcc4. Here, we show that the molecular ccrcc2 subtype defines highly angiogenic tumors, explaining the different therapeutic sensitivity of IMDC risk groups. It is enriched in IMDC goodrisk patients and can identify patients that are likely to respond to sunitinib or pazopanib within the intermediate-risk group. Immune signature is different between the 4 molecular subtypes, but not between IMDC risk groups.

- Sarcomatoid RCC have poor prognosis and poor outcome on sunitinib and pazopanib. Here, we show that ccRCC with $\geq 25 \%$ sarcomatoid component are molecularly different from those with $0 \%$ to $24 \%$, whereas there were no differences beween those with $0 \%$ and $1 \%$ to $24 \%$. Sarcomatoid ccRCC $(\geq 25 \%)$ were enriched for the ccrcc 4 subtype and had low angiogenic signature and poor outcome on sunitinib or pazopanib. In a small series treated with ICPIs, results were promising.

- Molecular subtypes therefore provide a maximum of information as a single generic biomarker. They are associated with angiogenic and immune signature, IMDC risk group, sarcomatoid dedifferentiation, and outcome on suntinib and pazopanib. They may be used for patient selection in the future, especially within the IMDC intermediate-risk group.

\section{Acknowledgments}

A. Verbiest, B. Beuselinck, and E. Lerut have received grants from FWO (Research Foundation-Flanders). Annelies Verbiest has received a grant from "Kom op tegen Kanker" (Stand up to Cancer), the Flemish cancer society.

\section{Disclosure}

B. Beuselinck reports consultancy fees from Amgen, Ipsen, Pfizer, and Novartis and research grants from Bristol-Myers Squibb and Ipsen. The remaining authors have stated that they have no conflicts of interest.

\section{Supplemental Data}

Supplemental tables accompanying this article can be found in the online version at https://doi.org/10.1016/j.clgc.2019.05.009.

\section{References}

1. Hsieh JJ, Purdue MP, Signoretti S, et al. Renal cell carcinoma. Nat Rev Dis Primers 2017; 3:17009.

2. Powles T, Albiges L, Staehler M, et al. Updated European Association of Urology Guidelines recommendations for the treatment of first-line metastatic clear cell renal cancer. Eur Urol 2018; 73:5.

3. Motzer RJ, Penkov K, Haanen J, et al. Avelumab plus axitinib versus sunitinib for advanced renal-cell carcinoma. N Engl J Med 2019; 380:1103-15.

4. Rini B, Huseni M, Atkins M, et al. Molecular correlates differentiate response to atezolizumab (atezo) + bevacizumab (bev) vs sunitinib (sun): Results from a phase III study (IMmotion151) in untreated metastatic renal cell carcinoma (mRCC). ESMO Annu Congress 2018:LBA31.

5. Rini BI, Plimack ER, Stus V, et al, KEYNOTE-426 Investigators. Pembrolizumab plus axitinib versus sunitinib for advanced renal-cell carcinoma. $N$ Engl J Med 2019; 380:1116-27.

6. National Institutes of Health; US National Library of Medicine. Lenvatinib/ everolimus or lenvatinib/pembrolizumab versus sunitinib alone as treatment of advanced renal cell carcinoma (CLEAR), Available at: https://clinicaltrialsgov/ct2/ show/NCT02811861. Accessed: December 18, 2018.

7. Motzer RJ, Tannir NM, McDermott DF, et al, CheckMate 214 Investigators. Nivolumab plus ipilimumab versus sunitinib in advanced renal-cell carcinoma. N Engl J Med 2018; 378:1277-90.

8. Heng DY, Xie W, Regan MM, et al. Prognostic factors for overall survival in patients with metastatic renal cell carcinoma treated with vascular endothelial growth factor-targeted agents: results from a large, multicenter study. J Clin Oncol 2009; 27:5794-9.

9. Rini BI, Hutson TE, Figlin RA, et al. Sunitinib in patients with metastatic renal cell carcinoma: clinical outcome according to International Metastatic Renal Cell Carcinoma Database Consortium Risk Group. Clin Genitourin Cancer 2018; 16:298-304.

10. Beuselinck B, Vano YA, Oudard S, et al. Prognostic impact of baseline serum Creactive protein in patients with metastatic renal cell carcinoma (RCC) treated with sunitinib. Clin Genitourin Cancer 2014; 114:81-9.

11. McDermott DF, Huseni MA, Atkins MB, et al. Clinical activity and molecular correlates of response to atezolizumab alone or in combination with bevacizumab versus sunitinib in renal cell carcinoma. Nat Med 2018; 24:749-57.

12. Beuselinck B, Job S, Becht E, et al. Molecular subtypes of clear cell renal cell carcinoma are associated with sunitinib response in the metastatic setting. Clin Cancer Res 2015; 21:1329-39.

13. Verbiest A, Couchy G, Job S, et al. Molecular subtypes of clear-cell renal cell carcinoma are prognostic for outcome after complete metastasectomy. Eur Urol 2018; 74:474-80.

14. The Cancer Genome Atlas Network. Comprehensive molecular characterization of clear cell renal cell carcinoma. Nature 2013; 499:43-9.

15. Brooks SA, Brannon AR, Parker JS, et al. ClearCode34: a prognostic risk predictor for localized clear cell renal cell carcinoma. Eur Urol 2014; 66:77-84.

16. Verbiest A, Couchy G, Job S, et al. Molecular subtypes of clear cell renal cell carcinoma are associated with outcome during pazopanib therapy in the metastatic setting. Clin Genitourin Cancer 2018; 16:e605-12.

17. Beuselinck B, Verbiest A, Couchy G, et al. Pro-angiogenic gene expression is associated with better outcome on sunitinib in metastatic clear-cell renal cell carcinoma. Act Oncol 2018; 57:498-508.

18. Harrell FE, Califf RM, Pryor DB, Lee KL, Rosati RA. Evaluating the yield of medical tests. JAMA 1982; 247:2543-6.

19. Beuselinck B, Lerut E, Wolter P, et al. Sarcomatoid dedifferentiation in metastatic clear cell renal cell carcinoma and outcome on treatment with anti-vascular endothelial growth factor receptor tyrosine kinase inhibitors: a retrospective analysis. Clin Genitourin Cancer 2014; 12:e205-14.

20. Golshayan AR, George S, Heng DY, et al. Metastatic sarcomatoid renal cell carcinoma treated with vascular endothelial growth factor-targeted therapy. J Clin Oncol 2009; 27:235-41. 


\section{Supplemental Data}

\begin{tabular}{|c|c|c|}
\hline \multirow{2}{*}{$\begin{array}{l}\text { Supplemental Table } 1 \\
\text { Characteristic }\end{array}$} & \multicolumn{2}{|c|}{$\begin{array}{l}\text { Characteristics of } 163 \text { Patients of } \\
\text { Whom IMDC Risk Group and Molecular } \\
\text { Data Were Available }\end{array}$} \\
\hline & & $\mathbf{N}$ \\
\hline Total & & 163 \\
\hline Male & & 113 \\
\hline \multicolumn{3}{|c|}{ First-line suntinib or pazopanib } \\
\hline Sunitinib & & 110 \\
\hline Pazopanib & & 35 \\
\hline Median age at start of first & line, $y$ & 63 \\
\hline \multicolumn{3}{|l|}{ IMDC risk group } \\
\hline Good & & 22 \\
\hline Intermediate & & 107 \\
\hline Poor & & 34 \\
\hline \multicolumn{3}{|l|}{ ccrcc1 to -4 subtype } \\
\hline ccrcc1 1 & & 49 \\
\hline ccrcc2 & & 70 \\
\hline $\operatorname{ccrcc} 3$ & & 10 \\
\hline $\operatorname{ccrcc} 4$ & & 34 \\
\hline \multicolumn{3}{|l|}{ Angio score } \\
\hline High & & 80 \\
\hline Low & & 83 \\
\hline \multicolumn{3}{|l|}{ T stage } \\
\hline $\mathrm{T} 1$ & & 28 \\
\hline T2 & & 19 \\
\hline T3 & & 109 \\
\hline $\mathrm{T} 4$ & & 4 \\
\hline Synchronous metastases & & 86 \\
\hline \multicolumn{3}{|l|}{ Fuhrman grade } \\
\hline 1 & & 0 \\
\hline 2 & & 6 \\
\hline 3 & & 53 \\
\hline 4 & & 104 \\
\hline \multicolumn{3}{|l|}{ Sarcomatoid dedifferentiation } \\
\hline$<25 \%$ & & 146 \\
\hline$\geq 25 \%$ & & 13 \\
\hline
\end{tabular}

Abbreviation: IMDC = International Metastatic RCC Database Consortium. 


\section{ARTICLE IN PRESS}

\section{Molecular Subtypes and Sarcomatoid Features in Clear-cell RCC}

Supplemental Tables 2 and 3. Differences in Angiogenic and Immune Gene Expression Between IMDC Risk Groups and ccrcc1 to ccrcc4 Molecular Subtypes.

\begin{tabular}{|c|c|c|c|c|c|c|c|c|c|}
\hline \multicolumn{2}{|c|}{ Supplemental Table 2} & \multicolumn{8}{|c|}{$\begin{array}{l}\text { Angiogenic Gene Expression is Higher for IMDC Good > Intermediate > Poor Risk and cerce2 }>\text { cerec3 }> \\
\text { cerce-1 > cerec4 Subtypes }\end{array}$} \\
\hline \multirow[b]{2}{*}{ Gene } & \multicolumn{4}{|c|}{ IMDC Risk Group } & \multicolumn{5}{|c|}{ ccrcc1 to -4 Molecular Subtype } \\
\hline & Good & Intermediate & Poor & $P$ Value & ccrcc1 & ccrcc2 & ccrcc3 & ccrce4 & $P$ Value \\
\hline VEGFA & 7.8 & 5.0 & 2.8 & $<.001$ & 3.2 & 8.5 & 2.0 & 2.5 & $<.0001$ \\
\hline VEGFC & 0.86 & 0.92 & 0.55 & .3 & 0.83 & 0.92 & 1.2 & 0.52 & .04 \\
\hline VEGFR1 & 3.3 & 1.8 & 0.10 & $<.001$ & 1.0 & 3.3 & 0.95 & 0.52 & $<.0001$ \\
\hline VEGFR2 & 1.6 & 0.87 & 0.38 & $<.001$ & 0.38 & 1.6 & 0.61 & 0.29 & $<.0001$ \\
\hline VEGFR3 & 1.1 & 0.83 & 0.40 & $<.001$ & 0.64 & 1.2 & 0.68 & 0.36 & $<.0001$ \\
\hline HIF1A & 0.81 & 0.63 & 0.64 & .3 & 0.81 & 0.64 & 2.2 & 0.50 & .008 \\
\hline HIF2A & 1.2 & 0.98 & 0.49 & $<.001$ & 0.67 & 1.3 & 0.95 & 0.39 & $<.0001$ \\
\hline PDGFRB & 1.5 & 1.4 & 1.1 & .07 & 1.4 & 1.6 & 1.6 & 0.89 & .008 \\
\hline ANGPTL4 & 88 & 70 & 35 & .05 & 72 & 103 & 2.6 & 27 & $<.0001$ \\
\hline
\end{tabular}

The largest difference is in expression of VEGFR2, the most important target of sunitinib and pazopanib $(+321 \%$ for IMDC Good vs. Poor and $+440 \%$ for ccrcc2 vs. ccrcc4). Abbreviation: IMDC = International Metastatic RCC Database Consortium.

\begin{tabular}{|c|c|c|c|c|c|c|c|c|c|}
\hline \multicolumn{2}{|c|}{ Supplemental Table 3} & \multicolumn{8}{|c|}{$\begin{array}{l}\text { Immune Gene Expression is } \\
\text { cCrecC2 > cerce1 and cerce3 }\end{array}$} \\
\hline \multirow[b]{2}{*}{ Gene } & \multicolumn{4}{|c|}{ IMDC Risk Group } & \multicolumn{5}{|c|}{ ccrcc1-4 Molecular Subtype } \\
\hline & Good & Intermediate & Poor & $P$ Value & ccrcc1 & ccrec2 & ccrcc3 & ccrec4 & $P$ Value \\
\hline$P D-L 1$ & 1.0 & 1.0 & 1.2 & .9 & 0.51 & 1.2 & 1.5 & 1.2 & .008 \\
\hline IFN-G & 2.4 & 2.8 & 2.0 & .5 & 1.4 & 2.9 & 1.2 & 6.3 & .0007 \\
\hline PD1 & 3.4 & 6.1 & 5.3 & .5 & 3.8 & 5.7 & 3.4 & 11.1 & .001 \\
\hline$P D-L 2$ & 1.3 & 1.6 & 1.3 & .9 & 0.78 & 1.8 & 1.4 & 2.2 & $<.0001$ \\
\hline LAG3 & 3.6 & 5.1 & 5.5 & .5 & 3.6 & 5.0 & 3.2 & 11 & $<.0001$ \\
\hline CD3G & 3.2 & 3.3 & 2.2 & .3 & 2.0 & 3.7 & 1.9 & 4.0 & .0004 \\
\hline CXCL13 & 4.3 & 8.4 & 9.5 & .3 & 4.9 & 8.2 & 2.5 & 34 & $<.0001$ \\
\hline CXCL9 & 2.7 & 3.3 & 2.6 & .9 & 1.9 & 4.3 & 1.8 & 10 & $<.0001$ \\
\hline CSF1R & 3.4 & 3.3 & 4.1 & .8 & 2.6 & 3.8 & 2.9 & 3.7 & .5 \\
\hline IL10 & 2.7 & 2.4 & 2.3 & 1 & 2.4 & 2.5 & 2.7 & 2.4 & .9 \\
\hline IL12a & 0.55 & 0.53 & 0.36 & .3 & 0.35 & 0.51 & 1.0 & 0.57 & .05 \\
\hline$T N F$ & 0.88 & 0.86 & 0.94 & 1 & 0.58 & 0.87 & 1.4 & 1.4 & .0006 \\
\hline TIM3 & 1.3 & 1.1 & 0.94 & .3 & 0.97 & 1.3 & 1.5 & 0.83 & .02 \\
\hline$C D 8 a$ & 4.8 & 6.3 & 4.3 & .5 & 3.6 & 7.4 & 2.1 & 10 & $<.0001$ \\
\hline
\end{tabular}

Median fold change is reported and compared with the Kruskal Wallis test. Abbreviation: IMDC = International Metastatic RCC Database Consortium. 
Supplemental Tables 5 and 6. Multivariable Cox regression for impact of sarcomatoid dedifferentiation on progression-free and overall survival on first-line sunitinib or pazopanib $(n=366)$. The following predictors were included in the models: sarcomatoid dedifferentiation ( $0 \%-24 \%$ vs. $\geq 25 \%$ ), IMDC (Good vs. Inter-

\begin{tabular}{|c|c|c|}
\hline \multirow[t]{2}{*}{ Supplemental Table 4} & \multicolumn{2}{|c|}{$\begin{array}{l}\text { Characteristics of } 366 \text { Patients Treated } \\
\text { With First-line Sunitinib or Pazopanib, } \\
\text { for Whom Percentage of Sarcomatoid } \\
\text { Dedifferentiation was Available }\end{array}$} \\
\hline & $\begin{array}{c}\text { Non-sarcomatoid } \\
(<25 \%) \\
n=344(\%)\end{array}$ & $\begin{array}{c}\text { Sarcomatoid } \\
(\geq 25 \%) \\
n=22(\%)\end{array}$ \\
\hline Median age at start, y & 64 & 61 \\
\hline Male & $228(66)$ & $16(73)$ \\
\hline \multicolumn{3}{|l|}{ First-line therapy } \\
\hline Sunitinib & $248(72)$ & $18(82)$ \\
\hline Pazopanib & $96(28)$ & $4(18)$ \\
\hline \multicolumn{3}{|l|}{ IMDC risk group } \\
\hline Good & $45(14)$ & 0 \\
\hline Intermediate & $195(60)$ & $9(43)$ \\
\hline Poor & $85(26)$ & $12(57)$ \\
\hline \multicolumn{3}{|l|}{ ccrcc 1 to -4 subtype } \\
\hline ccrcc1 1 & $44(30)$ & $3(23)$ \\
\hline ccrcc2 & $67(45)$ & $2(15)$ \\
\hline ccrcc3 & $9(6)$ & 0 \\
\hline ccrcc4 & $26(18)$ & $8(62)$ \\
\hline \multicolumn{3}{|l|}{ Angio score } \\
\hline High & 76 (52) & $3(23)$ \\
\hline Low & $70(48)$ & $10(77)$ \\
\hline \multicolumn{3}{|l|}{ T stage } \\
\hline $\mathrm{T} 1$ & $63(19)$ & $3(14)$ \\
\hline T2 & $64(20)$ & $3(14)$ \\
\hline T3 & $186(57)$ & $13(62)$ \\
\hline T4 & $13(4)$ & $2(10)$ \\
\hline Synchronous metastases & $163(47)$ & $15(68)$ \\
\hline \multicolumn{3}{|l|}{ Fuhrman grade } \\
\hline 1 & $5(1)$ & 0 \\
\hline 2 & $37(11)$ & 0 \\
\hline 3 & $143(42)$ & 0 \\
\hline 4 & $157(46)$ & $22(100)$ \\
\hline
\end{tabular}

Abbreviation: IMDC = International Metastatic RCC Database Consortium.

\section{Annelies Verbiest et al}

mediate vs. Poor), time of metastases (synchronous vs. metachronous), Fuhrman grade (< 4 vs. 4 ), T-stage (1-2 vs. $3-4)$, and age at start of first-line therapy. Molecular subtype and Angio score were not included, as these were only known for a minority of patients. Sarcomatoid dedifferentiation and IMDC risk group remained independent predictors of progression-free and overall survival.

\section{Supplemental Table 5 Progression-free Survival}

\begin{tabular}{l|c|c|c} 
Predictor & Hazard Ratio & $\mathbf{9 5 \%}$ Confidence Interval & \multicolumn{2}{|c}{$\boldsymbol{P}$ Value } \\
$\geq 25 \%$ sarcomatoid dedifferentiation & 2.9 & $1.8-4.5$ & $<.0001$ \\
\hline IMDC intermediate risk (vs. good) & 1.4 & $0.9-2.2$ & .1 \\
\hline IMDC poor risk (vs. good) & 2.3 & $1.5-3.7$ & .0003 \\
\hline
\end{tabular}

Abbreviation: IMDC = International Metastatic RCC Database Consortium. 
ARTICLE IN PRESS

\section{Molecular Subtypes and Sarcomatoid Features in Clear-cell RCC}

\section{Supplemental Table 6 Overall Survival}

\begin{tabular}{l|c|c|c} 
Predictor & Hazard Ratio & $\mathbf{9 5 \%}$ Confidence Interval & \multicolumn{2}{c}{$\boldsymbol{P}$ Value } \\
$\geq 25 \%$ sarcomatoid dedifferentiation & 2.3 & $1.4-3.7$ & .001 \\
\hline IMDC intermediate risk (vs. good) & 1.9 & $1.2-3.1$ & .009 \\
IMDC poor risk (vs. good) & 3.2 & $1.9-5.4$ & $<.0001$ \\
\hline
\end{tabular}

Abbreviation: IMDC = International Metastatic RCC Database Consortium.

Supplemental Tables 7 and 8 . Comparison of ccRCC with $0 \%$ and $1 \%$ to $24 \%$ Sarcomatoid Dedifferentiation.

\begin{tabular}{|c|c|c|c|}
\hline \multirow[t]{2}{*}{ Supplemental Table 7} & \multicolumn{3}{|c|}{$\begin{array}{l}\text { IMDC Risk Group and Angio Seore do Not Differ Between Tumors With 0\% Versus } 1 \% \text { to } 24 \% \text { Sarcomatoid } \\
\text { Dedifferentiation }\end{array}$} \\
\hline & $0 \%$, n (\%) & $1 \%-24 \%, n(\%)$ & $P$ Value \\
\hline IMDC risk group & & & .4 \\
\hline Good & $18(17)$ & $3(8)$ & \\
\hline Intermediate & $72(67)$ & $28(74)$ & \\
\hline Poor & $17(16)$ & 7 (18) & \\
\hline Median Angio score & -0.4 & -0.4 & .99 \\
\hline \multicolumn{4}{|c|}{ ccrcc 1 to -4 molecular subtype } \\
\hline ccrcc1 & $28(26)$ & $19(37)$ & Numbers too small for Fisher exact test \\
\hline ccrcc2 & $55(55)$ & $14(27)$ & \\
\hline $\operatorname{ccrcc} 3$ & $6(6)$ & $3(6)$ & \\
\hline ccrcc4 & $18(17)$ & $16(31)$ & \\
\hline
\end{tabular}

$P$ values calculated with the Fisher exact test (IMDC) and logistic regression (Angio score). Abbreviation: IMDC = International Metastatic RCC Database Consortium.

Supplemental Table 8 Expression of Angiogenesis Genes is Similar Between ceRCC With 0\% and 1\% to $24 \%$ Sarcomatoid Dedifferentiation, and Higher Compared With ceRCC With $\geq 25 \%$ Sarcomatoid Dedifferentiation

\begin{tabular}{l|c|c|c}
\multirow{2}{*}{ Gene } & \multicolumn{2}{|c}{ Sarcomatoid Component } \\
\cline { 2 - 4 } & $\mathbf{0} \%$ & $\mathbf{1 \% - 2 4 \%}$ & $\mathbf{2 5 \%}$ \\
\hline VEGFA & 5.7 & 4.7 & 2.7 \\
\hline VEGFC & 0.82 & 0.89 & 0.58 \\
\hline VEGFR1 & 1.8 & 1.9 & 0.46 \\
\hline VEGFR2 & 0.89 & 0.87 & 0.17 \\
VEGFR3 & 0.83 & 0.85 & 0.38 \\
\hline HIF1A & 0.71 & 0.83 & 0.47 \\
\hline HIF2A & 1.0 & 0.81 & 0.29 \\
\hline PDGFRB & 1.4 & 1.5 & 0.99 \\
\hline ANGPTL4 & 63 & 72 & 32 \\
\hline
\end{tabular}

Median fold change is reported.

Abbreviation: $\mathrm{ccRCC}=$ Clear-cell renal cell carcinoma

8.e4 Clinical Genitourinary Cancer Month 2019 
Supplemental Tables 9 and 10. The 3 IMDC Risk Groups are Distinctly Different; Therefore, Pooling of Intermediate and Poor Risk Should be Avoided When Possible.

\section{Supplemental Table 9 Comparison of IMDC Good-risk Palients With Pooled Intermediate/Poor Risk}

\begin{tabular}{|c|c|c|c|}
\hline & \multicolumn{2}{|c|}{ IMDC Risk Group } & \multirow[b]{2}{*}{$P$ Value } \\
\hline & Good & Intermediate/Poor & \\
\hline \multicolumn{4}{|c|}{$\begin{array}{l}\text { Outcome on first-line suntinib } \\
\text { or pazopanib }\end{array}$} \\
\hline $\mathrm{RR}, \%$ & 63 & 41 & .08 \\
\hline PFS, mos & 25 & 11 & .02 \\
\hline \multicolumn{4}{|l|}{ Angio score } \\
\hline High & $77 \%(17)$ & $45 \%(63)$ & .02 \\
\hline Low & $23 \%(5)$ & $55 \%(78)$ & \\
\hline \multicolumn{4}{|c|}{$\begin{array}{l}\text { Individual angiogenesis genes, } \\
\text { median fold change }\end{array}$} \\
\hline VEGFA & 7.8 & 4.3 & .01 \\
\hline VEGFC & 0.86 & .80 & .5 \\
\hline VEGFR1 & 3.3 & 1.59 & .004 \\
\hline VEGFR2 & 1.6 & .66 & .003 \\
\hline VEGFR3 & 1.1 & .72 & .02 \\
\hline HIFIA & 0.81 & .63 & .2 \\
\hline HIF2A & 1.2 & .79 & .01 \\
\hline PDGFRB & 1.5 & 1.4 & .2 \\
\hline ANGPTL4 & 88 & 63 & .2 \\
\hline \multicolumn{4}{|c|}{ ccrcc 1 to -4 molecular subtype } \\
\hline ccrcc1 & $14 \%(3)$ & $33 \%(46)$ & .002 \\
\hline ccrcc2 & $77 \%(17)$ & $38 \%(53)$ & \\
\hline ccrcc3 & $9 \%(2)$ & $6 \%(8)$ & \\
\hline ccrcc4 & 0 & $24 \%(34)$ & \\
\hline \multicolumn{4}{|c|}{ Sarcomatoid dedifferentiation } \\
\hline$<25 \%$ & $100 \%(21)$ & $91 \%(124)$ & .14 \\
\hline$\geq 25 \%$ & 0 & $9 \%(13)$ & \\
\hline
\end{tabular}

The differences are less pronounced and $P$ values are higher than upon comparison of the 3 IMDC risk groups separately.

Abbreviations: IMDC $=$ International Metastatic RCC Database Consortium; PFS $=$ progression-free survival; $\mathrm{RR}=$ response rate. 
ARTICLE IN PRESS

Molecular Subtypes and Sarcomatoid Features in Clear-cell RCC

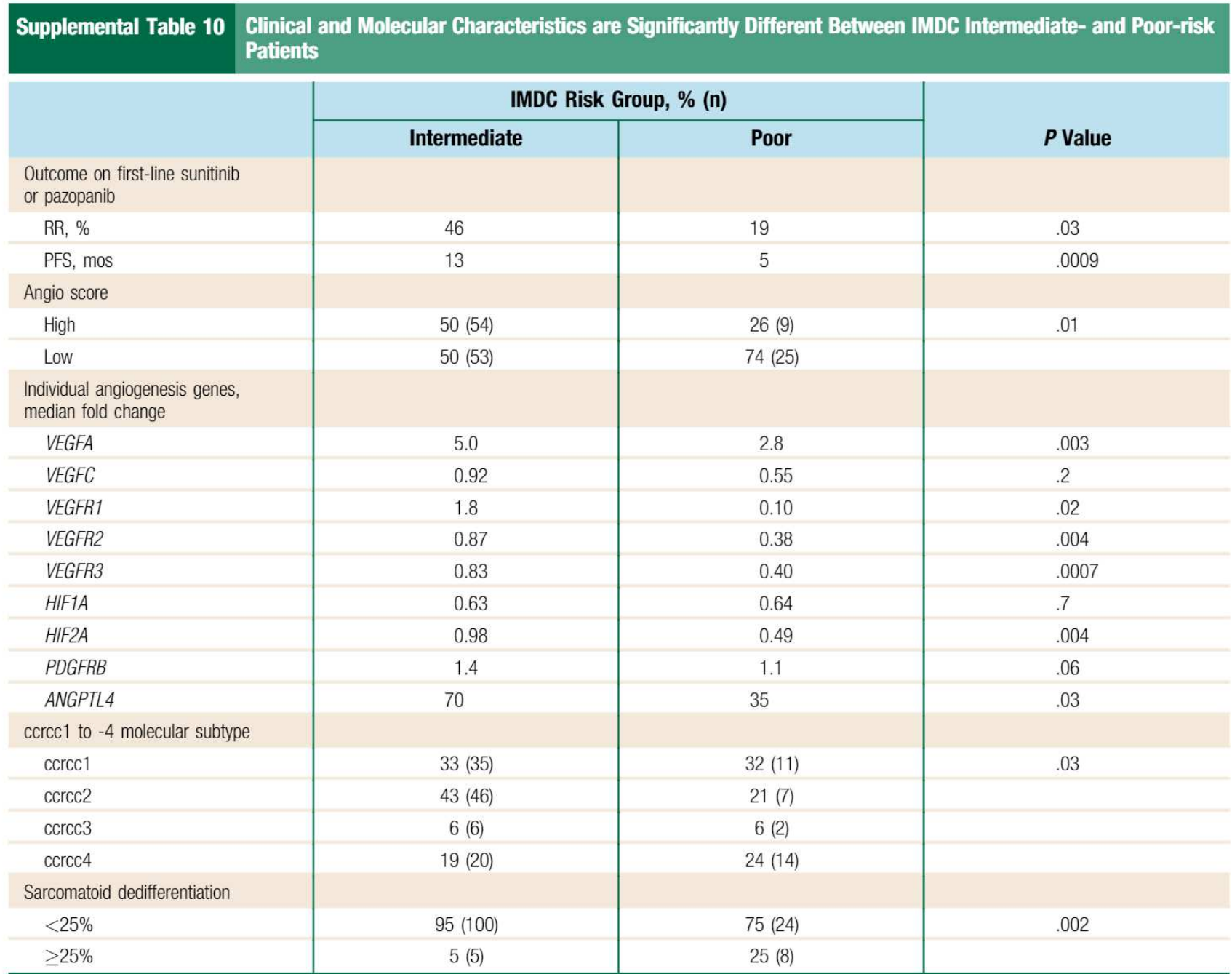

$P$ values were calculated with the Fisher exact test (Angio score, ccrcc1 to ccrcc4, sarcomatoid), logistic regression (RR), Kaplan-Meier method (PFS), and Mann Whitney $U$ test (fold change). Abbreviations: IMDC = International Metastatic RCC Database Consortium; PFS = progression-free survival; RR = response rate. 